\title{
CORDOTOMIA PERCUTÂNEA CERVICAL
}

\author{
ROLAND MÜKE * \\ AlCEU CORREIA**
}

Nos últimos 10 anos a cordotomia do quadrante ântero-lateral da medula espinhal desenvolveu-se em uma nova técnica, a interrupção percutânea das fibras condutoras da dor $7,11,12$. Apesar de ser a clássica cordotomia mediante laminectomia considerada como o mais indicado procedimento no tratamento das dores incuráveis do tronco e extremidades, era ela acompanhada de insuficiências, pois os sucessos no tratamento das dores no tórax, ombro e braço eram limitados. White e Sweet (1969) ${ }^{14}$, em 28 pacientes com afecções malignas dessas regiões, somente obtiveram cessação das dores em $54 \%$ dos casos, com uma mortalidade de $21 \%$, apesar de a intervenção em 27 casos ter sido apenas unilateral. Além disso nas operações visando à supressão de dores localizadas no hemicorpo inferior, ocorriam complicações como paresias e perturbações da micção com relativa freqüência ${ }^{2,9}$. Antes de tais intervenções era necessário considerar que, na maioria dos casos, a cordotomia é indicada em doentes em mau estado geral, para os quais é muito gravosa a carga adicional de uma operação, aumentando as possibilidades da ocorrência de tromboses, pneumonia e infecção urinária.

O desenvolvimento do método teria então que ter por meta principalmente alcançar também as fibras condutoras da dor dos segmentos cervicais e torácicos superiores e, ao mesmo tempo, controlar a interrupção destas fibras, permitindo imediato reconhecimento de complicações para evitá-las quando possivel. Este fim foi alcançado mediante a produção de lesões gradativamente aumentadas, por meio de um eletrodo colocado através da pele por via estereotática no quadrante ântero-lateral da medula espinhal, sem que seja necessária a visualização direta e, portanto, sem submeter o paciente a laminectomia.

\section{TE C I C A}

O paciente é colocado em posição supina em mesa operatória, de tal modo que o nível do corpo em relação à cabeça possa ser modificado. A cabeça é fixada com o auxílio de um suporte (Fig. 1). Na região lateral do pescoço, logo abaixo do processo mastoideu, sob anestesia local, é introduzida uma agulha de punção lombar. Sob controle de um monitor de T.V., em projeções ântero-posterior e lateral, atingese com a ponta da agulha a metade anterior do canal raqueano entre $o$ atlas $\mathrm{e}$

Do Departamento de Neurocirurgia da Clínica Neurológica da Universidade de Hamburgo, Alemanha Federal (Diretor: Prof. Dr. R. Kautzky): * Chefe de Clínica, Livre-Docente de Neurocirurgia; ** Neurocirurgião assistente. 
epistrofeus. Após a perfuração da dura-mater é atingido o espaço subaracnóideo, sendo a agulha fixada em um micromanipulador. A seguir injeta-se ar atmosférico em quantidades de 3 a $4 \mathrm{~cm}^{3}$, até se atingir um total de 10 a $20 \mathrm{~cm}^{3}$, com a finalidade de expôr o limite anterior da medula espinhal. Nesta fase do procedimento é importante que o corpo do paciente esteja de tal modo em relação à cabeça, que o ar não possa atingir o espaço subaracnoídeo intracraniano, o que pode ocasionar vômitos e cefaléia, prejudicando o decorrer da intervenção. Obtém-se, assim, uma mielografia gasosa cervical, que deve ser sem falhas. Empregando-se pequenas quantidades de ar, corre-se o perigo de se obter um nível ar/líquido cefalorraqueano (LCR) e, portanto, um falso contorno da medula, devido ao LCR que, neste caso, permanece na porção ventral do espaço subaracnoídeo cervical.

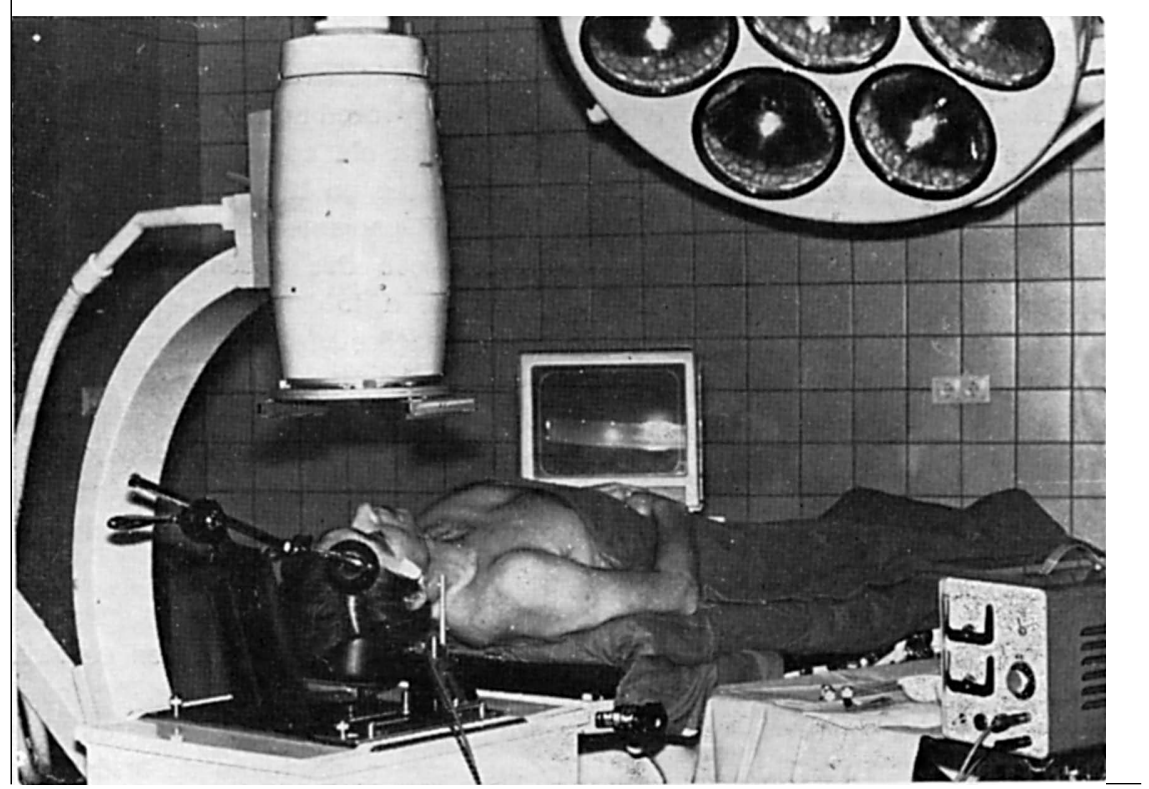

Fig. 1 - Cordotomia percutânea cervical à direita, vendo-se o paciente em posição operatória, o monitor de T.V., o suporte para a cabeca, o micromanipulador e o gerador de rádio-freqüência.

A seguir, com o micromanipulador, que permite movimentos milimétricos nos 3 planos, a ponta da agulha é dirigida ao quadrante ântero-lateral da medula, até que ela se projete 2 a $3 \mathrm{~mm}$ dorsal do contorno anterior da medula espinhal demonstrado pelo ar (Fig. 2). A ponta da agulha deve ser dirigida da parte ventral para a parte dorsal do canal espinhal, evitando-se que se movimente juntamente o ligamento denteado, o que ocasiona uma rotação da medula. Com a injeção de $0,5 \mathrm{~cm}^{3}$ de Pantopaque, obtém-se o contorno do ligamento denteado e, assim, a indicação da posição da ponta da agulha entre ligamento e limite anterior da medula. O tracto espinotalâmico situa-se perto do ligamento denteado, no quadrante antero-lateral. Sua localização e dimensão, apesar das várias publicações a respeito, em bases anatômicas e físiológicas, ainda não estão definidos com exatidão. Provavelmente existem variações individuais que poderiam explicar as diferentes concepcões dos autores 

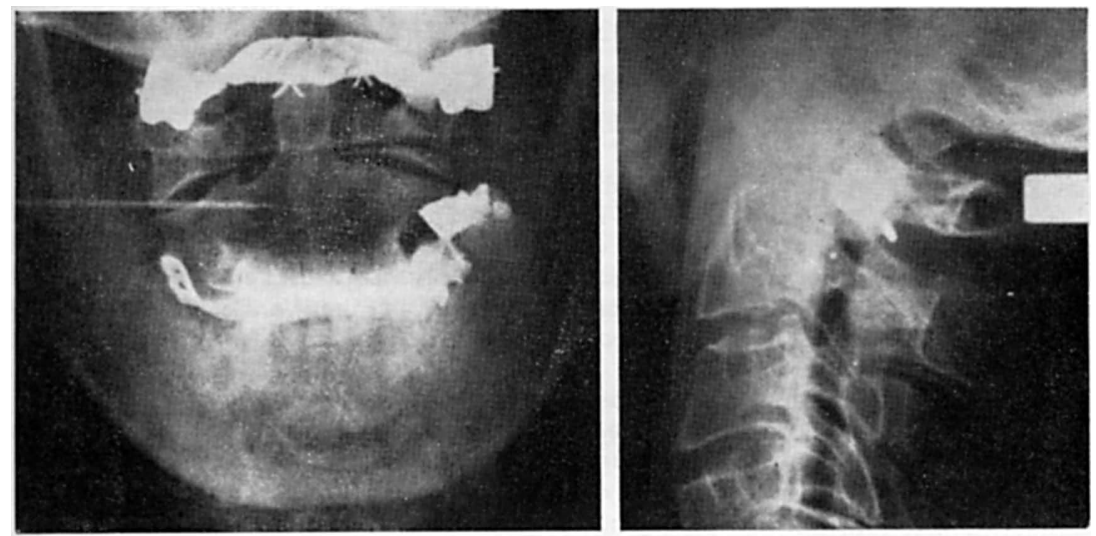

Fig. 2 - Agulha e eletrodo entre C1 e C2 no quadrante antero-lateral da medula espinhal, nas projecōes anteroposterior e lateral.

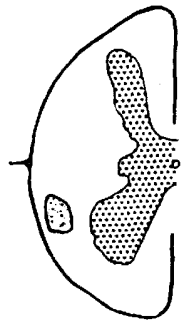

A.

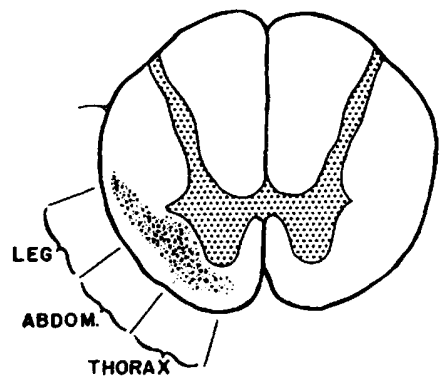

E.

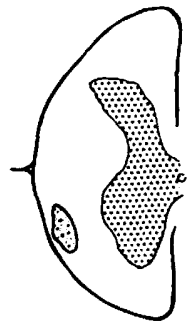

B.

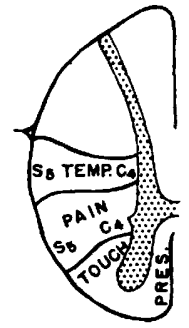

c.

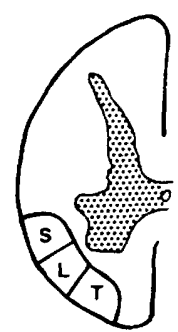

D.

Fig. 3 - Posição, tamanho e arranjo topográfico do tracto espinotalâmico segundo: (A) Gray 1942; (B) Ranson, 1920; (C) Foerster, 192\%; (D) Riley, 1943; (E) Hyndman e Van Epps, 1939; (F) Walker, 1940. Figura reproduzida, data venia, de J. C. White Conduction of pain in man. Arch. Neurol a. Psychiat. (Chicago) $71: 1,1954$. 
(Fig. 3). Através da agulha de punção é introduzido um ejetrodo, que ultrapassa a ponta da agulha em $4 \mathrm{~mm}$. A esta altura o paciente pode acusar dor, devido à penetração do eletrodo na pia-mater.

O eletrodo, que previamente é isolado por meio de um fino tubo de polietileno, com excessão de $2 \mathrm{~mm}$ em sua extremidade, é a seguir ligado a um gerador de rádio-freqüência. As lesões são feitas usando-se 20 Volts e $110 \mathrm{mAmp}$ e são aumentadas dradativamente depedendo da duração da corrente. $O$ gerador é regulado de tal modo que, em 5 segundos, obtém-se uma lesão elíptica de $1 \mathrm{~mm}$ no maior diâmetro e, em 30 segundos, uma lesão de $6 \mathrm{~mm}$. Estando o eletrodo em posição correta ocorre, no hemicorpo contralateral, uma analgesia ascendente e cessação imediata das dores. Uma lesão de $3 \mathrm{~mm}$ deve produzir uma analgesia até à região inguinal; uma lesão de $6 \mathrm{~mm}$ uma analgesia até os segmentos cervicais. Segundo nossa experiência, lesões de apenas 1 a $2 \mathrm{~mm}$ ( 5 a 10 segundos de corrente) podem interromper todo o tracto espinotalâmico.

Por outro lado, há casos em que se consegue facilmente uma lesão ascendente gradativa até uma determinada altura, tendo-se a seguir dificuldade em elevar o nível da analgesia. Estes resultados dependem com certeza diretamente da situaçāo do eletrodo em relação ao tracto espinotalâmico. Além da mielografia gasosa e do contorno do ligamento denteado obtido com Pantopaque, pode-se utilizar ainda para maior segurança as técnicas de impedância e estimulação elétrica da medula espinhal com a ponta do eletrodo. Na grande maioria dos casos, com alguma experiência, estes meios auxiliares não se fazem necessários. Durante a aplicação da corrente elétrica, prova-se continuamente a função motora do membro superior ipsilateral, solicitando que o paciente abra e feche a mão, com o braço elevado, de modo a se poder reconhecer, imediatamente, a ocorrência de lesão de fibras do tracto córticoespinhal. Após cada lesão examina-se cuidadosamente a função motora da extremidade ipsilateral.

Deve-se explicar ao paciente antecipadamente os detalhes da operação, solicitando sua cooperação que é dificultada quando se trata de pacientes em mau estadogeral ou com alterações psíquicas, ou ainda naqueles viciados em morfina. Apesar da fixação da cabeça podem ocorrer movimentos involuntários com modificação da situação da agulha, ou não serem autênticas as informações do doente quanto à analgesia e cessação das dores. A duração da intervenção é variável, entre 10 minutos e uma hora. Não se conseguindo efeito algum após uma hora, deve-se suspender e transferir a operação para outra data pois a concentração e cooperação do paciente em geral se torna insegura.

\section{MATERIAL E RESULTADOS}

Desde maio de 1971 foram realizadas, em 59 pacientes, 72 cordotomias percutâneas cervicais, sendo 60 unilaterais e 6 bilaterais. A cordotomia foi repetida em 7 vezes; em 5 casos em virtude da queda do nível da analgesia no pós-operatório e, em 2 casos, por não ter sido obtido efeito na primeira intervenção.

$\mathrm{Na}$ maior parte dos casos foram operados doentes com neoplasias (13 carcinomas bronquiais e pulmonares; 7 carcinomas de recto; 4 carcinomas de útero; 3 carcinomas de mama; 3 sarcomas ósseos metastáticos; 2 melanoblastomas metastáticos; 2 carcinomas do sigma; 2 carcinomas de bexiga; 1 carcinoma da laringe; 1 carcinoma do colon; 1 fibrosarcoma; 1 plasmocitoma; 1 caso de moléstia de Hodgkin metastático; 1 metástase de tumor do glomo carotídeo). A medida que se obteve maior experiência, a cordotomia foi indicada para doentes com dores incuráveis de natureza não maligna (6 casos de dores-fantasmas; 4 casos de pacientes com dores após operações em hérnias discais; 1 paciente com cicatrizes espinhais após excisão de um lipoma; 1 ganglioneuroma espinhal; 1 caso de herpes zoster; 1 paciente com dores após uma paraplegia traumática; 1 paciente com dores após fratura complicada de tíbia; 1 paciente com intenso e incurável prurido anal).

A cessação das dores com analgesia foi obtida em 61 das 72 cordotomias; a cessação das dores sem analgesia em 2; a diminuição das dores em 3; em 6 casos 
nenhum efeito foi observado. O nivel da analgesia foi alcançado: em 16 vezes até o 2.0 segmento cervical; em 13 vezes do $3 .^{\circ}$ ao $8 .^{\circ}$ segmento cervical; em 27 vezes do 1.0 ao 10.0 segmento torácico; em 5 vezes abaixo do 10.0 segmento torácico. Com excessão de dois, todos estes pacientes apresentaram completa insensibilidade à dor nos segmentos caudais ao limite da analgesia. Dos dois casos em que não houve analgesia total, em um observou-se analgesia no tórax e membro inferior com poupança do abdome e, em outro, analgesia no tronco e hipoalgesia na extremidade inferior.

Complicações - Dos 72 pacientes operados, 8 apresentaram paresias de extremidades, 4 vezes durante a operação e 4 vezes no pós-operatório. Das primeiras, uma foi acentuada e permanente, 3 foram discretas sendo que uma apresentou melhora e duas desapareceram. Das 4 paresias pós-operatórias, 3 foram passageiras; a evolução no $40^{\circ}$ caso não pôde ser apreciada uma vez que o paciente faleceu devido a outra complicação. As paresias intraoperatórias são devidas a lesões determinadas em local errado; movimentos da cabeça durante aplicações da corrente elétrica são as principais causas desta complicação. Pacientes com enfermiddes na parte superior do tórax, no ombro e no pescoço, apesar da fixação, têm dificuldades em manter a cabeça na posição correta; o mesmo ocorre em pacientes em mau estado-geral ou com alterações psiquicas, cuja cooperação seja deficiente. As paresias pós-operatórias têm como causa o edema ou distúrbios na vascularização na região lesada. As paresias permanentes nas nossas primeiras 72 cordotomias ocorreram em 3 casos $(4,1 \%)$ e com certeza essa percentagem diminui à medida que se obtém maior experiência.

Quatro pacientes apresentaram dificuldades passageiras na micção; outros dois apresentaram problemas de micção em virtude da enfermidade primária, não correlacionados com a intervenção. Três pacientes do sexo masculino e de meia idade (um com dores fantasmas e dois com dores após operações em hérnias discais) apresentaram perturbações na potência sexual, apesar de ter sido feita, nos três casos, cordotomia unilateral.

Em 4 casos ocorreu depressão respiratória: em 3 após cordotomias bilaterais nos quais a analgesia foi elevada até os segmentos cervicais; no $4 .^{\circ}$ caso após uma cordotomia unilateral em paciente cuja funçāo pulmonar do lado das dores estava deprimida devido a uma toracoplastia. A depressão respiratória não se apresentou imediatamente e sim no $2 .^{\circ}$ e $3 .^{\circ}$ dias pós-operatórios e apresentou evolução variável, com fases nas quais os pacientes necessitaram de respiração artificial, alternando com períodos de respiração espontânea. Destes 4 casos, dois não apresentaram outras complicações; um apresentou paresia de extremidade intraoperatória; um apresentou paresia de extremidade pós-operatória. Em nenhum deles o estado de consciência se alterou devido ao problema respiratório.

Registramos 5 éxitos letais $(6,9 \%)$, sendo 3 após cordotomias bilaterais. A enfermidade primária foi com certeza responsável pelos óbitos de 4 pacientes, se bem que em três casos a depressăo respiratória sem dúvida acelerou a evolução. Um paciente faleceu em decorrência direta da cordotomia, com problema respiratório.

As queixas pós-operatórias mais freqüentes foram cefaléias, provavelmente devido à penetração de ar nos ventrículos cerebrais e neuralgias no $2 .^{\circ}$ segmento cervical no lado da punção, ambas leves e passageiras. Vários doentes apresentaram uma sindrome de Horner ipsilateral. Quase todos os pacientes acusaram, por ocasião dos primeiros passos, no $1^{\circ}$ ou $2 .^{\circ}$ dia pós-operatório, uma ataxia ipsilateral, que desapareceu em todos os casos em 2 a 3 dias. A permanência no hospital é, em regra, curta, de 3 a 5 dias. Quando foi possível e na dependência da enfermidade primária, os pacientes foram considerados como aptos a reassumir suas profissões.

\section{COMENTARIOS}

Em nossos 59 pacientes obtivemos, com a cordotomia percutânea cervical, abolição das dores em 53 casos $(90 \%)$. Nas 72 cordotomias feitas nesses 59 
pacientes, ocorreram, como acidentes, paresias permanentes em 3 casos $(4,1 \%)$, crises respiratórias em 4 pacientes e 5 óbitos $(6,9 \%)$. Nos 28 casos em que a analgesia era necessária somente para a metade inferior do hemicorpo obteve-se sucesso em 26, sem complicações permanentes (Quadro 1). Os resultados melhoraram à medida que se obteve maior experiência. Mesmo Rosomoff e col. ${ }^{11,12}$ que já realizaram mais de 1.000 cordotomias percutâneas, observaram, no primeiro grupo de 35 pacientes, resultados favoráveis com cessação das dores em apenas 24 casos; ulteriormente em 184 cordotomias em 100 pacientes, conseguiram o efeito desejado em 92\% dos casos, assinalando, como complicações, 7 paresias temporárias e uma permanente, 7 perturbações passageiras da miç̧ão e 4 óbitos. Outros autores obtiveram resultados menos favoráveis $3,4,6,7$.

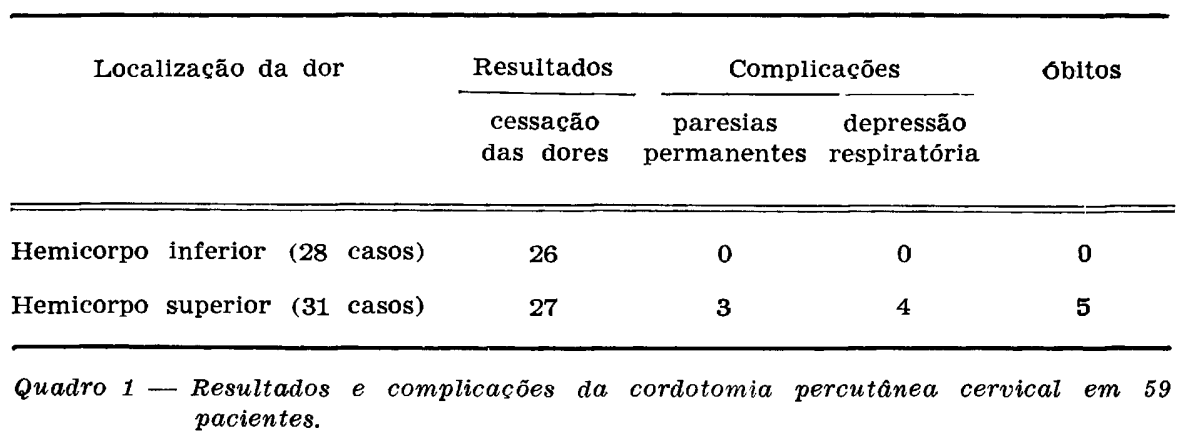

Os resultados da cordotomia clássica mediante laminectomia são, em geral, piores. Nathan ${ }^{9}$, do Serviço de McKissok, referiu que em 104 pacientes, dos quais 68 operados pelo próprio McKissok, somente foi obtida total cessação das dores em 58 casos (52\%); em 28 casos houve melhora; em 17 discreta melhora; em 8 nenhum efeito útil. Bohm ${ }^{2}$ referiu, em 1960, 73 casos dos quais somente em $50 \%$ foi obtida cessação permanente das dores (a avaliação da evolução foi feita em diferentes estádios do pós-operatório). Este autor assinalou, em seu material, 11 paresias ( 5 acentuadas e permanentes, 2 moderadas e 4 discretas) e 13 distúrbios da micção, dos quais 6 permanentes. Ogle e col. ${ }^{10}$ publicaram em 1956 os resultados de 81 cordotomias cervicais, com 13 paresias das quais 12 foram passageiras, 11 retenções de urina sendo 9 passageiras, porém com uma mortalidade de 13,5\% (11 casos, dos quais 5 após cordotomias bilaterais). Os dados falam por si mesmos, pois estas operações foram realizadas em renomadas clínicas.

Mesmo que seja possível, com maior experiência e com métodos micro. cirúrgicos, melhorar os resultados da cordotomia clássica, o método percutâneo continuará a ser mais vantajoso. O sucesso na cessação das dores é mais seguro, os resultados quanto às dores localizadas no ombro e no braço são melhores, o traumatismo operatório é menor, o tempo de permanência pós-operatória no hospital é mais curto. 
Problemáticos, também para o método percutâneo, são os casos nos quais seja necessária a analgesia até os segmentos cervicais. Apesar de empregar técnica exata, o perigo de depressão respiratória se faz sempre presente ${ }^{8,12}$. É provável que lesões bilaterais nos quadrantes ântero-laterais da medula espinhal cervical interrompam estímulos da periferia para o centro respiratório, cujo tônus se tornaria menor, exigindo maior concentração de $\mathrm{CO}_{2}$ no sangue para sua estimulação. O paciente "esquece" de respirar. Esta perturbação seria passageira, podendo, no entanto, permanecer durante algumas semanas. Alguns autores admitem que lesões no quadrante ântero-lateral interrompem também fibras motoras para o diafragma e para a musculatura intercostal ${ }^{1}$. Uma possibilidade para contornar este risco, seria combinar uma cordotomia percutânea cervical de um lado com uma mesencefalotomia ou talamotomia do outro lado, ou mediante uma cordotomia percutânea por via anterior ${ }^{5}$ em segmentos cervicais mais inferiores (em C5/C6, por exemplo). Sabendo que com o eletrodo em posição absolutamente exata é possível interromper todo o tracto espinotalâmico com uma lesão elíptica de 1 a $2 \mathrm{~mm}$ no diâmetro maior, existe outra possibilidade de evitar o problema respiratório, uma vez que este somente aparece após lesões maiores.

Nos casos em que as dores se localizam no ombro e no braço, deve-se procurar focalizar o tracto cuidadosamente com auxílio de estimulação elétrica. O risco de depressão respiratória se torna também menor quando se realiza a segunda intervenção no lado oposto ao da primeira com intervalos maiores ( 8 a 14 dias). Complicações respiratórias de caráter perigoso surgem somente em uma parte dos casos. Exames pré-operatórios da função respiratória não trazem infelizmente informações sobre a tendência dos pacientes para esta complicação ${ }^{12}$.

O problema das recidivas não pode, com base em nossa experiência atual, ser debatido nesta publicação, pois o tempo de seguimento pós-operatório de nossos casos é ainda curto. Uma avaliação cuidadosa se faz extremamente necessária, principalmente em relação à indicação da operação nos casos em que as dores não sejam provocadas por neoplasias. Destes pacientes, 3 apresentaram graves problemas de potência sexual, embora todos se sintam satisfeitos com a cessação das dores. Com a ocorrência de recidivas a situação se modificaria totalmente.

\section{R ES U M O}

Com bases em experiência pessoal, são dadas informações sobre a técnica, as vantagens, os resultados e os problemas da cordotomia percutânea cervical. Este método representa nítida evolução para melhor, em comparação com a cordotomia clássica feita após laminectomia. Entre as vantagens salientamse: o melhor sucesso na cessação das dores; os melhores resultados obtidos nas dores localizadas no ombro e no braço; menor traumatismo operatório; a diminuição do tempo de permanência dos pacientes no hospital. Constituem ainda problemas para o método percutâneo os casos que exigem cordotomias 
bilaterais nos quais seja necessário elevar a analgesia até os segmentos cervicais e os casos nos quais a cooperação dos pacientes seja insuficiente. Em 72 cordotomias percutâneas realizadas foi obtida a cessação das dores em $90 \%$ dos casos, com 3 paresias $(4,1 \%)$ e mortalidade de $6,9 \%$.

\section{Z US A M M E N F A S U N G}

\section{Die percutane cervicale Chordotomie}

Aufgrund eigener Erfahrungen wird über die percutane cervicale Chordotomie berichtet. Technisches Vorgehen, Verteile und Probleme der Methode werden erläutert. Die percutane Chordotomie ist ein deutlicher Fortschritt gegenüber der bisher geübten offenen Chordotomie. Als Vorteile zählen: Die Schmerzausschaltung ist sicherer, Komplikationen sind leichter zu vermeiden, Schulter-Arm-Schmerzen besser erfa $\beta$ bar, der Eingriff ist weniger belastend, die postoperative Krankenhausliegezeit kürzer. Probleme bringen immer noch die bilateralen hohen Chordotomien aufgrund von möglichen Atemfunktionsstörungen, ferner mitunter die Kooperation mit den Patienten. Bei 72 selbst durchgeführten Chordotomien wurde in 90\% die gewünschte Schmerzfreiheit erreicht, dabei kam es in 4,1\% zu Paresen, die Mortalität betrug $6,9 \%$.

\section{S U M M A R Y}

\section{Percutaneous cervical cordotomy}

This is a report concerned with the procedure of percutaneous cervical cordotomy. Technical details, advantages and problems of the operation are discussed. Percutaneous cordotomy definitively is to be prefered over open cordotomy. Advantages are: control is more definite, complications are easier to avoid, shoulder-arm pain is better treatble, the procedure is generally less taxing, postoperative hospitalisation is shortened. Problematical are bilateral cordotomies with high levels or cervical analgesia, due to disturbances of respiratory function and furthermore the cooperation of the patient. In 72 percutaneous cordotomies we controlled pain in $90 \%, 41 \%$ experienced paresis of different localisation, mortality was $6,9 \%$.

\section{REFERENCIA S}

1. BELMUSTO, L.; WOLDRING, S. \& OWENS, G. - Localisation and patterns of potentials of the respiratory pathway in the cervical spinal cord in the dog. J. Neurosurg. 22:272, 1965.

2. BOHM, E. - Cordotomy for intractable pain due to malignant disease. Acta psychiatr. scand. 35:145, 1960.

3. BURTON, M. - Recent results with percutaneous cordotomy. Mayo Clin. Proc. $45: 689,1970$. 
4. FOX, J. L. - Percutaneous stereotaxic cordotomy. Acta Neurochir. 18:309, 1968.

5. FOX, J. L. \& GREEN, R. C. - Stereotaxic surgery using a television guidance system. Acta Neurochir. 21:31, 1969.

6. MUKE, R. - Schmerzausschaltung durch percutane zervikale Chordotomie. Deutsch. Med. Woch. 32:1169, 1972.

7. Mullan, S.; HeKMatPanaH, G.; DOBben, G. \& BECKMAN, F. - Percutaneous, intramedullary cordotomy utilizing the unipolar anodal electrolytic lesion. J. Neurosurg. 22:588, 1965.

8. MULLAN, S. \& HOSOBUCHI, X. - Respiratory hazards of high cervical percutaneous cordotomy. J. Neurosurg. 28:291, 1965.

9. NATHAN, P. W. - Results of antero-lateral cordotomy for pain in cancer. J. Neurol. Neurosurg. Psychiatr. 26:355, 1963.

10. OGLE, W. S.; FRENCH, L. A. \& PEYTON, W. T. - Experiences with high cervical cordotomy. J. Neurosurg. 13:81, 1956.

11. ROSOMOFF, H. L.; CARROL, F.; BROWN, J. \& SHEPTAK, P. - Percutaneous radiofrequency cervical cordotomy: technique. J. Neurosurg. 23:639, 1965.

12. ROSOMOFF, H. L.; SHEPTAK, P. \& CARROL, F. - Modern pain relief: percutaneous cordotomy. J.A.M.A. 196:482, 1966.

13. ROSOMOFF, H. L.; KRIEGER, A. J. \& KUPERMAN, A. S. - Effects of percutaneous cervical cordotomy on pulmonary function. J. Neurosurg. 31:620, 1969.

14. WHITE, J. C. \& SWEET, M. W. - Pain and the Neurosurgeon. Carles C. Thomas Publisher, Springfield (Illinois), 1969, págs. 678-712.

Neurochirurgische Abieilung - Universitätskrankenhaus Eppendorf — 2000 Hamburg 20 - Martinistrasse 52-W. Deutschland. 